\title{
LMI BASED STABILITY AND STABILIZATION OF SECOND-ORDER LINEAR REPETITIVE PROCESSES
}

\author{
Pawel Dabkowski, Krzysztof Gałkowski, Biswa Datta and Eric Rogers
}

\begin{abstract}
This paper develops new results on the stability and control of a class of linear repetitive processes described by a second-order matrix discrete or differential equation. These are developed by transformation of the secondorder dynamics to those of an equivalent first-order descriptor state-space model, thus avoiding the need to invert a possibly ill-conditioned leading coefficient matrix in the original model.
\end{abstract}

Key Words: LMI, discrete and differential second-order linear repetitive processes, ill-conditioning, descriptor systems.

\section{INTRODUCTION}

Second order linear control systems arise in a wide variety of practical applications involving, for example, vibrating structures, power systems, economics, and computer networks. One obvious way to solve a control problem for a linear second-order system is to transform the model to first-order statespace form and then use any of the well known and

\footnotetext{
Manuscript received December 24, 2008; accepted April 14, 2009.

P. Dabkowski and K. Galkowski are with the Institute of Physics, Nicolaus Copernicus University Torun, Poland (e-mail: p.dabkowski@fizyka.umk.pl).

$\mathrm{K}$. Galkowski is also with the Institute of Control and Computation Engineering, University of Zielona Gora, Zielona Gora, Poland (e-mail: k.galkowski@issi.uz.zgora.pl).

B. Datta is with Departmemt of Mathematical Sciences and Vibration and Acoustic Center of the College of Engineering and Engineering Technology, Northern Illinois University, Dekalb, Illinois, 600115, U.S.A. (e-mail: dattab@math.niu.edu).

E. Rogers is with School of Electronics and Computer Science, University of Southampton, SO17 1BJ, U.K. (e-mail: etar@ecs.soton.ac.uk).

This work is partially supported by the Nicolaus Copernicus University Torun grant $351-F$ and the Ministry of Science and Higher Education in Poland under the project N N514 293235. The work of Biswa Datta was partially supported by NSF Grant DMS-0505784 and US Department of Education Grant P116Z080102 to Vibration and Acoustic Center of NIU.
}

tested computational methods. Unfortunately, such reduction requires explicit computation of the inverse of the leading coefficient matrix, which could be numerically problematic due, for example, to possible illconditioning of this matrix or the computational cost involved. For example, in vibration control analysis, this matrix, termed the mass matrix, is often diagonal and therefore can be ill-conditioned whenever some (or all) of the diagonal entries are small (see [1]).

Another area where such problems can arise is in the application of the Crank-Nicholson discretization scheme to partial differential equations (PDEs) [2]. Here the resulting model coefficient matrices are often tridiagonal but the inverse of the leading coefficient one may not have this computationally attractive property. A similar situation arises for first-order descriptor systems, where the coefficient matrix on the left-hand side may be very close to singular or left-multiplying the model by the inverse of this matrix involves the loss of other essential problem features. To overcome such difficulties research has been focussed in recent years on developing methods for second-order state-space models that do not require explicit computation of a matrix inverse.

As a result of such research, there as been much progress on the solution of control related problems for systems described by second-order state-space models. Examples here include stability, feedback stabilization, partial pole placement, robust pole placement, and model order reduction. These solutions have 
been developed, in the main, by either first converting to an equivalent descriptor system or proceeding directly with the coefficient matrices of the secondorder state-space model [3-7]. The latter approach has the further advantage that any special structure, such as sparsity, in the coefficient matrices, which often arises in practical applications, can be preserved and exploited in the computations associated with numerical examples. Such problems can also arise in 2D linear systems where, for example, repetitive processes have found application in modeling spatio-temporal dynamics such as large flexible structures [8].

The unique characteristic of a repetitive, or multipass [9], process is a series of sweeps, termed passes, through a set of dynamics defined over a fixed finite duration known as the pass length. On each pass an output, termed the pass profile, is produced which acts as a forcing function on, and hence contributes to, the dynamics of the next pass profile. This, in turn, leads to the unique control problem in that the output sequence of pass profiles generated can contain oscillations that increase in amplitude in the pass-to-pass direction.

Physical examples of repetitive processes include long-wall coal cutting and metal rolling operations [10]. Also in recent years applications have arisen where adopting a repetitive process setting for analysis has distinct advantages over alternatives. Examples of these so-called algorithmic applications include classes of iterative learning control schemes [11]. In this last case, for example, use of the repetitive process setting provides the basis for the development of highly reliable and efficient solution algorithms and in the former it provides a stability theory which, unlike alternatives, provides information concerning an absolutely critical problem in this application area, i.e. the trade-off between convergence and the learnt dynamics.

Attempts to control these processes using standard (or 1D) systems theory/algorithms fail (except in a few very restrictive special cases) precisely because such an approach ignores their inherent 2D systems structure, i.e. information propagation occurs from pass-to-pass and along a given pass and also the initial conditions are reset before the start of each new pass. To remove these deficiencies, a rigorous stability theory has been developed [10] based on an abstract model of the dynamics in a Banach space setting which includes a very large class of processes with linear dynamics and a constant pass length as special cases. Also the results of applying this theory to a range of sub-classes, including those considered here, have been reported [10]. This stability theory consists of the distinct concepts of asymptotic stability and stability along the pass respectively where the former is a necessary condition for the latter.
In this paper we develop new results on the stability and control of linear repetitive processes where the pass-to-pass updating is governed by a matrix linear second-order discrete or differential equation with possible numerical ill-conditioning. The major outcome is Linear Matrix Inequality (LMI)-based algorithms for stability testing and control law design, including the case when there is uncertainty associated with the process dynamics.

Throughout this paper, the null matrix and the identity matrix with the required dimensions are denoted by 0 and $I$, respectively. Moreover, $M>0(<0)$ denotes a real symmetric positive (respectively negative) definite matrix, and $\star$ denotes a block matrix entry in a symmetric matrix.

\section{BACKGROUND}

Let $\alpha<\infty$ denote the pass length and use an integer subscript $k \geq 0$ to denote the pass number or index. Then the most basic discrete linear repetitive process state-space model [10] has the following form over $0 \leq$ $p \leq \alpha-1, k \geq 0$,

$$
\begin{aligned}
x_{k+1}(p+1) & =A x_{k+1}(p)+B u_{k+1}(p)+B_{0} y_{k}(p) \\
y_{k+1}(p) & =C x_{k+1}(p)+D u_{k+1}(p)+D_{0} y_{k}(p) .
\end{aligned}
$$

Here on pass $k, x_{k}(p) \in \mathbb{R}^{n}$ is the state vector, $y_{k}(p) \in \mathbb{R}^{m}$ is the pass profile vector, and $u_{k}(p) \in \mathbb{R}^{r}$ is the vector of control inputs. The boundary conditions (i.e. the pass state initial vector sequence and the initial pass profile) are

$$
\begin{aligned}
x_{k+1}(0) & =d_{k+1}, \quad k \geq 0 \\
y_{0}(p) & =f(p), \quad 0 \leq p \leq \alpha-1
\end{aligned}
$$

where the $n \times 1$ vector $d_{k+1}$ has known constant entries and $f(p)$ is an $m \times 1$ vector whose entries are known functions of $p$.

In a differential linear repetitive process [10] the along the pass dynamics are governed by a linear matrix differential equation and, with the along the pass variable denoted by $t$, the most basic state-space model has the following form over $0 \leq t \leq \alpha, k \geq 0$,

$$
\begin{aligned}
& \dot{x}_{k+1}(t)=A x_{k+1}(t)+B u_{k+1}(t)+B y_{k}(t) \\
& y_{k+1}(t)=C x_{k+1}(t)+D u_{k+1}(t)+D_{0} y_{k}(t)
\end{aligned}
$$

where all notation is the same as the discrete case, except that the initial pass profile is now taken as $y_{0}(t)=f(t)$, where the entries in $f(t)$ are known functions over $0 \leq t \leq \alpha$. 
The stability theory [10] for linear repetitive processes is based on an abstract model in a Banach space setting which includes a wide range of such processes as special cases, including both cases considered in this work. In terms of their dynamics it is the pass-to-pass coupling (noting again their unique feature) which is critical in the analysis of linear repetitive processes. This is of the form $y_{k+1}=L_{\alpha} y_{k}$, where $y_{k} \in E_{\alpha}\left(E_{\alpha}\right.$ a Banach space with norm $\|\cdot\|)$ and $L_{\alpha}$ is a bounded linear operator mapping $E_{\alpha}$ into itself. (In the cases considered here $L_{\alpha}$ are discrete and differential linear systems convolution operators respectively.)

Asymptotic stability, i.e. BIBO stability over the fixed finite pass length $\alpha>0$, requires the existence of finite real scalars $M_{\alpha}>0$ and $\lambda_{\alpha} \in(0,1)$ such that $\left\|L_{\alpha}^{k}\right\| \leq$ $M_{\alpha} \lambda_{\alpha}^{k}, k \geq 0$, (where $\|\cdot\|$ denotes the induced operator norm). For the discrete and differential linear repetitive processes considered in this work it has been shown elsewhere (see, for example, Chapter 3 of [10]) that this property holds if, and only if, all eigenvalues of the matrix $D_{0}$ have modulus strictly less than unity, written here as $r\left(D_{0}\right)<1$ where $r(\cdot)$ denotes the spectral radius of its matrix argument.

Suppose that a process described by either of the state-space models considered here is asymptotically stable and also that the input sequence applied $\left\{u_{k+1}\right\}_{k}$ converges strongly as $k \rightarrow \infty$ (i.e. in the sense of the norm on the underlying function space) to $u_{\infty}$. Then the strong limit $y_{\infty}:=\lim _{k \rightarrow \infty} y_{k}$ is termed the limit profile corresponding to this input sequence. For the discrete process, it can be shown that the limit profile is given by

$$
\begin{aligned}
x_{\infty}(p+1)= & \left(A+B_{0}\left(I-D_{0}\right)^{-1} C\right) x_{\infty}(p) \\
& +\left(B+B_{0}\left(I-D_{0}\right)^{-1} D\right) u_{\infty}(p) \\
y_{\infty}(p)= & \left(I-D_{0}\right)^{-1} C x_{\infty}(p) \\
& +\left(I-D_{0}\right)^{-1} D u_{\infty}(p) \\
x_{\infty}(0)= & d_{\infty}
\end{aligned}
$$

where $d_{\infty}$ is the strong limit of the sequence $\left\{u_{k}\right\}$.

In physical terms, this result states that under asymptotic stability the repetitive dynamics can, after a 'sufficiently large' number of passes have elapsed, be replaced by those of a $1 \mathrm{D}$ discrete linear system. In particular, this property demands that the amplifying properties of the coupling between successive pass profiles are completely suppressed after a sufficiently large number of passes have elapsed. This fact has clear implications in terms of the control of these processes.

The fact that the pass length is finite means that the limit profile may have unacceptable along the pass dynamics. For example, consider the case when $A=-0.5$,
$B=1, B_{0}=0.5+\beta, C=1, D=0, D_{0}=0$ where $\beta$ is a real scalar. This example is asymptotically stable since $D_{0}=0$ and the state matrix of the resulting limit profile state-space model is $\beta$. Hence the limit profile is unstable unless $|\beta|<1$. Clearly this is not acceptable in many cases.

The limit profile for the differential case is

$$
\begin{aligned}
\dot{x}_{\infty}(t)= & \left(A+B_{0}\left(I-D_{0}\right)^{-1} C\right) x_{\infty}(t) \\
& +\left(B+B_{0}\left(I-D_{0}\right)^{-1} D\right) u_{\infty}(t) \\
y_{\infty}(t)= & \left(I-D_{0}\right)^{-1} C x_{\infty}(t) \\
& +\left(I-D_{0}\right)^{-1} D u_{\infty}(t) \\
x_{\infty}(0)= & d_{\infty}
\end{aligned}
$$

In order to avoid cases where asymptotic stability results in an unstable limit profile, the obvious route is to demand the BIBO property for all possible values of the pass length (mathematically this can be analyzed by letting $\alpha \rightarrow \infty$ ). This is the stability along the pass property which (in abstract model terms) requires the existence of finite real scalars $M_{\infty}>0$ and $\lambda_{\infty} \in(0,1)$, independent of $\alpha$, such that $\left\|L_{\alpha}^{k}\right\| \leq M_{\infty} \lambda_{\infty}^{k}, k \geq 0$. For discrete processes described by (1) and (2), it has been shown elsewhere that this requires

- $r\left(D_{0}\right)<1$ (asymptotic stability),

- $r(A)<1$, and

- $r(G(z))<1, \forall|z|=1$, where $G(z)=C(z I-A)^{-1}$ $B_{0}+D_{0}$.

In the case of processes described by (3), and the corresponding conditions are

- $r\left(D_{0}\right)<1$ (asymptotic stability),

- all eigenvalues of the matrix $A$ have strictly negative real parts, and

- $r(G(s))<1, \forall s: \operatorname{Re} s \geq 0$, where $G(s)=C(s I-A)^{-1} B_{0}+D_{0}$.

Note here that (1D) stability of the state matrix $A$ is also only necessary for stability along the pass, as the simple example above for the discrete case demonstrates.

For the processes considered here stability along the pass is independent of the boundary conditions assumed in this paper and hence they will not be explicitly stated in the theorems to follow that give the main results. Note, however, that the form of the boundary conditions is critical to the stability properties of linear repetitive processes. In particular, it can be shown [10] that if the state initial vector on each pass is a function of points along the previous pass then this alone can cause instability. 
In terms of stability analysis and control law design, the most productive route is via a $2 \mathrm{D}$ Lyapunov equation [10] characterization of stability along the pass which, in turn, arises from a Lyapunov function interpretation. The starting point is to note that any candidate Lyapunov function needs to capture the 'energy' associated with information propagation both along the pass and from pas-to-pass. The function used here for the discrete case is

$$
V(k, p)=x_{k+1}^{T}(p) W_{1} x_{k+1}(p)+y_{k}^{T}(p) W_{2} y_{k}(p)
$$

where $W_{1}>0$ and $W_{2}>0$, with associated increment

$$
\begin{aligned}
\Delta V(k, p)= & x_{k+1}^{T}(p+1) W_{1} x_{k+1}(p+1) \\
& +y_{k+1}^{T}(p) W_{2} y_{k+1}(p) \\
& -x_{k+1}^{T}(p) W_{1} x_{k+1}(p) \\
& -y_{k}^{T}(p) W_{2} y_{k}(p)
\end{aligned}
$$

Then we have the following result via the 2D Lyapunov equation.

Theorem 1 ([10]). A discrete linear repetitive process described by (1) with Lyapunov function (6) is stable along the pass if

$$
\Delta V(k, p)<0
$$

for all $0 \leq p \leq \alpha-1, k \geq 0$.

Now we have the following results which are central to the analysis in this paper.

Theorem 2 ([10]). A discrete linear repetitive process described by (1) is stable along the pass if $\exists$ matrices $P>0$ and $Q>0$ such that

$$
\left[\begin{array}{ccc}
-P+Q & 0 & \hat{A}_{1}^{T} P \\
0 & -Q & \hat{A}_{2}^{T} P \\
P \hat{A}_{1} & P \hat{A}_{2} & -P
\end{array}\right]<0
$$

where

$$
\hat{A}_{1}=\left[\begin{array}{cc}
A & B_{0} \\
0 & 0
\end{array}\right], \quad \hat{A}_{2}=\left[\begin{array}{cc}
0 & 0 \\
C & D_{0}
\end{array}\right] .
$$

Theorem 3 ([12]). A differential linear repetitive process described by (3) is stable along the pass if $\exists$ matrices $P_{1}>0$ and $P_{2}>0$ such that

$$
\begin{gathered}
{\left[\begin{array}{ccc}
-P_{2} & P_{2} C & P_{2} D_{0} \\
C^{T} P_{2} & A^{T} P_{1}+P_{1} A & P_{1} B_{0} \\
D_{0}^{T} P_{2} & B_{0}^{T} P_{1} & -P_{2}
\end{array}\right]} \\
<0
\end{gathered}
$$

The discrete processes considered in the remainder of this paper are, with the notation as above, described by the following state-space model which is secondorder in the pass-to-pass direction

$$
\begin{aligned}
x_{k+1}(p+1)= & A x_{k+1}(p)+B u_{k+1}(p) \\
& +B_{10} y_{k+1}(p)+B_{00} y_{k}(p) \\
D_{2} y_{k+2}(p)= & C_{1} x_{k+1}(p)+D_{1} u_{k+1}(p) \\
& +D_{10} y_{k+1}(p)+D_{00} y_{k}(p)
\end{aligned}
$$

It is also necessary to extend the boundary conditions of (2) by adding

$$
y_{1}(p)=f_{1}(p), \quad p=0,1, \ldots,(\alpha-1)
$$

where $f_{1}(p)$ is an $m \times 1$ vector whose entries are known functions of $p$. This model (11) can be transformed to first-order form by introducing

$$
Y_{k}(p)=\left[\begin{array}{c}
y_{k}(p) \\
y_{k+1}(p)
\end{array}\right]
$$

to obtain

$$
\begin{aligned}
x_{k+1}(p+1)= & A x_{k+1}(p)+B u_{k+1}(p) \\
& +\hat{B}_{0} Y_{k}(p) \\
\Psi_{0} Y_{k+1}(p)= & \hat{C} x_{k+1}(p)+\hat{D} u_{k+1}(p) \\
& +\hat{D}_{0} Y_{k}(p)
\end{aligned}
$$

where

$$
\begin{aligned}
\hat{B_{0}} & =\left[\begin{array}{ll}
B_{00} & B_{10}
\end{array}\right], \quad \hat{C}=\left[\begin{array}{c}
0 \\
C_{1}
\end{array}\right] \\
\hat{D} & =\left[\begin{array}{c}
0 \\
D_{1}
\end{array}\right], \quad \hat{D_{0}}=\left[\begin{array}{cc}
0 & I_{m} \\
D_{00} & D_{10}
\end{array}\right] \\
\Psi_{0} & =\left[\begin{array}{cc}
I_{m} & 0 \\
0 & D_{2}
\end{array}\right] .
\end{aligned}
$$


The corresponding model in the differential case is

$$
\begin{aligned}
\dot{x}_{k+1}(t)= & A x_{k+1}(t)+B u_{k+1}(t) \\
& +B_{10} y_{k+1}(t)+B_{00} y_{k}(t) \\
D_{2} y_{k+2}(t)= & C_{1} x_{k+1}(t)+D_{1} u_{k+1}(t) \\
& +D_{10} y_{k+1}(t)+D_{00} y_{k}(t)
\end{aligned}
$$

and it is necessary to add

$$
y_{1}(t)=f_{1}(t), \quad 0 \leq t \leq \alpha
$$

to the boundary conditions where $f_{1}(t)$ is an $m \times 1$ vector whose entries are known functions of $t$. In first-order form we have for this case we introduce

$$
Y_{k}(t)=\left[\begin{array}{c}
y_{k}(t) \\
y_{k+1}(t)
\end{array}\right]
$$

to obtain

$$
\begin{aligned}
\dot{x}_{k+1}(t) & =A x_{k+1}(t)+B u_{k+1}(t)+\hat{B_{0}} Y_{k}(t) \\
\Psi_{0} Y_{k+1}(t) & =\hat{C} x_{k+1}(t)+\hat{D} u_{k+1}(t)+\hat{D_{0}} Y_{k}(t)
\end{aligned}
$$

Obviously, left-multiplying the second equation of (13) or (15) as appropriate by the matrix $\Psi_{0}^{-1}$ yields the repetitive process model of (1) or (3) respectively and then existing results can be applied. In terms of applications, however, problems will arise if this matrix is ill-conditioned. In this paper we develop methods that do not require this inversion and the possible ill-conditioning associated with constructing the inverse. Note, however that these do not extend to the case where the matrix $D_{2}$ is singular. To deal with this case it is necessary to use further results for singular linear systems, see, for example, [13].

\section{ANALYSIS}

Consider the discrete case. Then we cannot directly apply Theorem 2 to obtain a condition for stability along the pass of a process described by (11) since this requires the numerical inversion of the matrix $D_{2}$. Instead, we have the following result.

Theorem 4. A discrete linear repetitive process described by (13) is stable along the pass if $\exists$ matrices $Y>0$ and $Z>0$ such that

$$
\left[\begin{array}{ccc}
-Y+Z & 0 & Y \tilde{A}_{1}^{T} \\
0 & -Z & Y \tilde{A}_{2}^{T} \\
\tilde{A}_{1} Y & \tilde{A}_{2} Y & -\Psi Y \Psi^{T}
\end{array}\right]<0
$$

where

$$
\tilde{A}_{1}=\left[\begin{array}{cc}
A & \hat{B}_{0} \\
0 & 0
\end{array}\right], \quad \tilde{A}_{2}=\left[\begin{array}{cc}
0 & 0 \\
\hat{C} & \hat{D}_{0}
\end{array}\right]
$$

and

$$
\Psi=\left[\begin{array}{cc}
I_{n} & 0 \\
0 & \Psi_{0}
\end{array}\right] .
$$

Proof. First left-multiply the second equation in (13) by $\Psi_{0}^{-1}$ and apply Theorem 2 to the result. The proof is then completed by application of obvious congruence transforms and change of variables.

Theorem 5. A differential linear repetitive process described by (15) is stable along the pass if $\exists$ matrices $Y_{1}>0$ and $Z_{1}>0$ such that

$$
\left[\begin{array}{ccc}
-\Psi_{0} Z_{1} \Psi_{0}^{T} & \hat{C} Y_{1} & \hat{D}_{0} Z_{1} \\
Y_{1} \hat{C}^{T} & Y_{1} A^{T}+A Y_{1} & \hat{B}_{0} Z_{1} \\
Z_{1} \hat{D}_{0}^{T} & Z_{1} \hat{B}_{0}^{T} & -Z_{1}
\end{array}\right]<0
$$

Proof. First Left-multiply the second equation of (15) by $\Psi_{0}^{-1}$ and apply Theorem 3 to the result. Next, left and right-multiply the result of this last step by $\operatorname{diag}\left(\Psi_{0} P_{2}^{-1}, P_{1}^{-1}, P_{2}^{-1}\right)$ to obtain (17).

If stability along the pass is not present for a given example then it will clearly be necessary to introduce regulation action to guarantee this property. Moreover, given the critical role of the pass-to-pass updating, it follows that any control law must have a contribution from the previous two passes here plus current pass state or pass profile activated action. Here we consider a law of the form

$$
\begin{aligned}
u_{k+1}(p) & =K_{1} x_{k+1}(p)+\hat{K}_{2} y_{k}(p)+\hat{K}_{3} y_{k+1}(p) \\
& =K\left[\begin{array}{c}
x_{k+1}(p) \\
Y_{k}(p)
\end{array}\right]
\end{aligned}
$$

for the discrete case with differential counterpart

$$
\begin{aligned}
u_{k+1}(t) & =K_{1} x_{k+1}(t)+\hat{K}_{2} y_{k}(t)+\hat{K}_{3} y_{k+1}(t) \\
& =K\left[\begin{array}{c}
x_{k+1}(t) \\
Y_{k}(t)
\end{array}\right]
\end{aligned}
$$

where

$$
K=\left[\begin{array}{lll}
K_{1} & \hat{K}_{2} & \hat{K}_{3}
\end{array}\right]=\left[\begin{array}{ll}
K_{1} & K_{2}
\end{array}\right] .
$$


The pass profile vector is the process output and here it assumed that noise corruption and other disturbances are negligible. Moreover, ill-conditioning of the matrix $D_{2}$ is not a problem here in practical implementation of the control law. Also the current pass state vector in this stabilization law will, in general, require an observer.

The controlled process in the discrete case is described by

$$
\begin{aligned}
x_{k+1}(p+1)= & A_{\text {new }} x_{k+1}(p)+B_{n 1} y_{k+1}(p) \\
& +B_{n 2} y_{k}(p) \\
D_{2} y_{k+2}(p)= & C_{n} x_{k+1}(p)+D_{n 1} y_{k+1}(p) \\
& +D_{n 2} y_{k}(p)
\end{aligned}
$$

and in the differential case by

$$
\begin{aligned}
\dot{x}_{k+1}(t)= & A_{\text {new }} x_{k+1}(t)+B_{n 1} y_{k+1}(t) \\
& +B_{n 2} y_{k}(t) \\
D_{2} y_{k+2}(t)= & C_{n} x_{k+1}(t)+D_{n 1} y_{k+1}(t) \\
& +D_{n 2} y_{k}(t)
\end{aligned}
$$

where

$$
\begin{aligned}
A_{\text {new }} & =A+B K_{1}, \quad B_{n 1}=B_{10}+B \hat{K}_{3} \\
B_{n 2} & =B_{00}+B \hat{K}_{2}, \quad C_{n}=C_{1}+D_{1} K_{1} \\
D_{n 1} & =D_{10}+D_{1} \hat{K}_{3}, \quad D_{n 2}=D_{00}+D_{1} \hat{K}_{2}
\end{aligned}
$$

or, more compactly,

$$
\begin{gathered}
x_{k+1}(p+1)=A_{\text {new }} x_{k+1}(p)+B_{\text {new }} Y_{k}(p) \\
\Psi_{0} Y_{k+1}(p)=C_{\text {new }} x_{k+1}(p)+D_{\text {new }} Y_{k}(p)
\end{gathered}
$$

and

$$
\begin{aligned}
\dot{x}_{k+1}(t) & =A_{\text {new }} x_{k+1}(t)+B_{\text {new }} Y_{k}(t) \\
\Psi_{0} Y_{k+1}(t) & =C_{\text {new }} x_{k+1}(t)+D_{\text {new }} Y_{k}(t)
\end{aligned}
$$

respectively, where

$$
\begin{aligned}
& B_{\text {new }}=\left[\begin{array}{ll}
B_{n 2} & B_{n 1}
\end{array}\right]=\hat{B}_{0}+B K_{2} \\
& C_{\text {new }}=\left[\begin{array}{c}
0 \\
C_{n}
\end{array}\right]=\hat{C}+\hat{D} K_{1} \\
& D_{\text {new }}=\left[\begin{array}{cc}
0 & I_{m} \\
D_{n 2} & D_{n 1}
\end{array}\right]=\hat{D}_{0}+\hat{D} K_{2} .
\end{aligned}
$$

In the discrete case we now have the following result for stability along the pass of the controlled process together with a formula for computing the control law matrix.

Theorem 6. Suppose that a control law of the form (18) is applied to a discrete linear repetitive process described by (13). Then the resulting controlled process is stable along the pass if $\exists$ matrices $Y>0, Z>0$, and $\hat{N}=\left[\begin{array}{ll}N_{1} & N_{2}\end{array}\right]$ such that

$$
\left[\begin{array}{ccc}
-Y+Z & \star & \star \\
0 & -Z & \star \\
\tilde{A}_{1} Y+\tilde{B}_{1} \hat{N} & \tilde{A}_{2} Y+\tilde{B}_{2} \hat{N} & -\Psi Y \Psi^{T}
\end{array}\right]
$$

$<0$

where

$$
\tilde{B}_{1}=\left[\begin{array}{l}
B \\
0
\end{array}\right], \quad \tilde{B}_{2}=\left[\begin{array}{l}
0 \\
\hat{D}
\end{array}\right]
$$

If this condition holds, a stabilizing control law matrix $K$ is given by

$$
K=\hat{N} Y^{-1}
$$

Proof. The proof is a direct consequence of interpreting the LMI of Theorem 4 in terms of the case considered here with $K Y=\hat{N}$ and application of routine manipulations.

For the differential case we have the following result.

Theorem 7. Suppose that a control law of the form (19) is applied to a differential repetitive process described by (15). Then the resulting controlled process is stable along the pass if $\exists$ matrices $Y>0, Z>0, N$, and $M$ such that

$$
\begin{aligned}
& {\left[\begin{array}{ccc}
Y A^{T}+N^{T} B^{T} & & \star \\
+A Y+B N & & \\
\left(\hat{B}_{0} Z+B M\right)^{T} & -Z & \star \\
\hat{C} Y+\hat{D} N & \hat{D}_{0} Z+\hat{D} M & -\Psi_{0} Z \Psi_{0}^{T}
\end{array}\right]} \\
& <0
\end{aligned}
$$


If this condition holds, a stabilizing control law matrix $K$ is given by

$$
\begin{aligned}
& K_{1}=N Y^{-1} \\
& K_{2}=M Z^{-1}
\end{aligned}
$$

Proof. This follows analogous steps to the proof of the last result and hence the details are omitted here.

\section{STABILITY AND STABILIZATION OF UNCERTAIN PROCESSES}

In the most cases the model matrices are subject to uncertainty and only the nominal model is known. The standard route in robust control to deal with this case is to assume an uncertainty model and here we use the norm bounded type of uncertainty under which the discrete linear repetitive process of (11) takes the form

$$
\begin{aligned}
x_{k+1}(p+1)= & (A+\Delta A) x_{k+1}(p) \\
& +(B+\Delta B) u_{k+1}(p) \\
& +\left(B_{10}+\Delta B_{10}\right) y_{k+1}(p) \\
& +\left(B_{00}+\Delta B_{00}\right) y_{k}(p) \\
D_{2} y_{k+2}(p)= & \left(C_{1}+\Delta C_{1}\right) x_{k+1}(p) \\
& +\left(D_{1}+\Delta D_{1}\right) u_{k+1}(p) \\
& +\left(D_{10}+\Delta D_{10}\right) y_{k+1}(p) \\
& +\left(D_{00}+\Delta D_{00}\right) y_{k}(p)
\end{aligned}
$$

and in the differential case

$$
\begin{aligned}
\dot{x}_{k+1}(t)= & (A+\Delta A) x_{k+1}(t) \\
& +(B+\Delta B) u_{k+1}(t) \\
& +\left(B_{10}+\Delta B_{10}\right) y_{k+1}(t) \\
& +\left(B_{00}+\Delta B_{00}\right) y_{k}(t) \\
D_{2} y_{k+2}(t)= & \left(C_{1}+\Delta C_{1}\right) x_{k+1}(t) \\
& +\left(D_{1}+\Delta D_{1}\right) u_{k+1}(t) \\
& +\left(D_{10}+\Delta D_{10}\right) y_{k+1}(t) \\
& +\left(D_{00}+\Delta D_{00}\right) y_{k}(t)
\end{aligned}
$$

For analysis purposes these models can be rewritten as

$$
\begin{aligned}
x_{k+1}(p+1)= & (A+\Delta A) x_{k+1}(p) \\
& +(B+\Delta B) u_{k+1}(p) \\
& +\left(\hat{B_{0}}+\widehat{\Delta B_{0}}\right) Y_{k}(p) \\
\Psi_{0} Y_{k+1}(p)= & (\hat{C}+\widehat{\Delta C}) x_{k+1}(p) \\
& +(\hat{D}+\widehat{\Delta D}) u_{k+1}(p) \\
& +\left(\hat{D_{0}}+\widehat{\Delta D_{0}}\right) Y_{k}(p)
\end{aligned}
$$

and

$$
\begin{aligned}
\dot{x}_{k+1}(t)= & (A+\Delta A) x_{k+1}(t) \\
& +(B+\Delta B) u_{k+1}(t) \\
& +\left(\hat{B}_{0}+\widehat{\Delta B_{0}}\right) Y_{k}(t) \\
\Psi_{0} Y_{k+1}(t)= & (\hat{C}+\widehat{\Delta C}) x_{k+1}(t) \\
& +(\hat{D}+\widehat{\Delta D}) u_{k+1}(t) \\
& +\left(\hat{D}_{0}+\widehat{\Delta D_{0}}\right) Y_{k}(t)
\end{aligned}
$$

respectively, where

$$
\begin{aligned}
& \widehat{\Delta B_{0}}=\left[\begin{array}{ll}
\Delta B_{00} & \Delta B_{10}
\end{array}\right], \quad \widehat{\Delta C}=\left[\begin{array}{c}
0 \\
\Delta C_{1}
\end{array}\right] \\
& \widehat{\Delta D}=\left[\begin{array}{c}
0 \\
\Delta D_{1}
\end{array}\right], \\
& \widehat{\Delta D_{0}}=\left[\begin{array}{cc}
0 & I_{m} \\
\Delta D_{00} & \Delta D_{10}
\end{array}\right]
\end{aligned}
$$

and the rest of notation follows that of the previous section.

Now introduce the following notation.

$$
\begin{aligned}
& {\left[\begin{array}{lll}
A+\Delta A & \hat{B}_{0}+\widehat{\Delta B_{0}} & B+\Delta B \\
\hat{C}+\widehat{\Delta C} & \hat{D_{0}}+\widehat{\Delta \hat{D}_{0}} & \hat{D}+\widehat{\Delta D}
\end{array}\right]} \\
& \quad=\Xi+\Delta \Xi .
\end{aligned}
$$

Also it assumed that we can write

$$
\Delta \Xi=H F E=\left[\begin{array}{l}
H_{1} \\
H_{2}
\end{array}\right] F\left[\begin{array}{ll}
E_{1} & E_{2}
\end{array}\right]
$$

where $H, E_{1}, E_{2}$ are given matrices of compatible dimensions, and $F$ is unknown matrix which satisfies $\|F\|<1$, or $F^{T} F<I$. 


\subsection{Stability}

4.1.1 Discrete processes.

Introduce the following notation

$$
\begin{aligned}
\Delta \tilde{A}_{1} & =\left[\begin{array}{cc}
\Delta A & \widehat{\Delta B}_{0} \\
0 & 0
\end{array}\right] \\
\Delta \tilde{A}_{2} & =\left[\begin{array}{cc}
0 & 0 \\
\widehat{\Delta C} & \widehat{\Delta D_{0}}
\end{array}\right] \\
\Delta \tilde{B}_{1} & =\left[\begin{array}{c}
\Delta B \\
0
\end{array}\right] \\
\Delta \tilde{B}_{2} & =\left[\begin{array}{c}
0 \\
\widehat{\Delta D}
\end{array}\right]
\end{aligned}
$$

and

$$
\hat{H}_{1}=\left[\begin{array}{c}
H_{1} \\
0
\end{array}\right], \quad \hat{H}_{2}=\left[\begin{array}{c}
0 \\
H_{2}
\end{array}\right] .
$$

Then we have the following result by direct application of an existing LMI based stability condition.

Theorem 8. A discrete linear repetitive process described by (30) with uncertainty modeled as (32) is stable along the pass if, $\exists$ matrices $P>0$, and $Q>0$, such that

$$
(\widetilde{A}+\Delta \widetilde{A})^{T} P(\widetilde{A}+\Delta \widetilde{A})+\mathbb{Q}<0
$$

where

$$
\mathbb{Q}=\left[\begin{array}{cc}
P-Q & 0 \\
0 & Q
\end{array}\right], \quad \tilde{A}=\left[\begin{array}{cc}
\tilde{A_{1}} & \tilde{A}_{2}
\end{array}\right]
$$

and

$$
\Delta \widetilde{A}=\left[\begin{array}{ll}
\Delta \tilde{A}_{1} & \Delta \tilde{A}_{2}
\end{array}\right]
$$

To remove the uncertain term $F$ in this last result (which means that it is numerically intractable) we apply the elimination lemma [14] to obtain the following result.

Theorem 9. The condition of Theorem 8 holds if, and only if, $\exists$ a scalar $\varepsilon>0$ and matrices $P>0$, and $Q>0$, such that

$$
\begin{aligned}
& {\left[\begin{array}{cc}
-\Psi P^{-1} \Psi^{T}+\varepsilon \hat{H} \hat{H}^{T} & \tilde{A} \\
\tilde{A}^{T} & \mathbb{Q}+\varepsilon^{-1} \hat{E}_{1}^{T} \hat{E}_{1}
\end{array}\right]} \\
& <0
\end{aligned}
$$

where

$$
\hat{H}=\left[\begin{array}{ll}
\hat{H}_{1} & \hat{H}_{2}
\end{array}\right], \quad \hat{E}_{1}=\operatorname{diag}\left(E_{1}, E_{1}\right)
$$

Now we have the following result whose proof follows after standard algebraic manipulations that are omitted here.

Theorem 10. A discrete linear repetitive process described by (30) with uncertainty modeled as (32) is stable along the pass if $\exists$ a scalar $\varepsilon>0$, and matrices $Y>0$, and $Z>0$, such that

$$
\left[\begin{array}{cccc}
-\Psi Y \Psi^{T} & \tilde{A} \hat{Y} & \varepsilon \hat{H} & 0 \\
\hat{Y} \tilde{A}^{T} & \mathbb{Z} & 0 & \hat{Y} \hat{E}_{1}^{T} \\
\varepsilon \hat{H}^{T} & 0 & -\varepsilon I & 0 \\
0 & \hat{E}_{1} \hat{Y} & 0 & -\varepsilon I
\end{array}\right]<0
$$

where

$$
\hat{Y}=\left[\begin{array}{ll}
Y & 0 \\
0 & Y
\end{array}\right], \quad \mathbb{Z}=\left[\begin{array}{cc}
-Y+Z & 0 \\
0 & -Z
\end{array}\right]
$$

\subsubsection{Differential processes.}

We require the following well known result.

Lemma 11 ([15]). Let $\Sigma_{1}, \Sigma_{2}$ be real matrices of appropriate dimensions. Then for any matrix $\mathcal{F}$ satisfying $\mathcal{F}^{T} \mathcal{F} \leq I$ and a scalar $\varepsilon>0$ the following inequality holds

$$
\Sigma_{1} \mathcal{F} \Sigma_{2}+\Sigma_{2}^{T} \mathcal{F}^{T} \Sigma_{1}^{T} \preceq \varepsilon^{-1} \Sigma_{1} \Sigma_{1}^{T}+\varepsilon \Sigma_{2}^{T} \Sigma_{2}
$$

Applying Lemma 11 to the result of Theorem 5 interpreted in terms of the uncertain process (31) gives, after routine algebraic manipulations, the following result.

Theorem 12. A differential linear repetitive process described by (31) is stable along the pass if $\exists$ a scalar $\varepsilon>0$, and matrices $Y_{1}>0$, and $Z_{1}>0$, such that

$$
\left[\begin{array}{ccccc}
\Xi_{1} & \hat{C} Y_{1} & \hat{D}_{0} Z_{1} & 0 & 0 \\
Y_{1} \hat{C}^{T} & \Xi_{2} & \hat{B}_{0} Z_{1} & Y_{1}^{T} E_{1}^{T} & Y_{1}^{T} E_{1}^{T} \\
Z_{1} \hat{D}_{0}^{T} & Z_{1} \hat{B}_{0}^{T} & -Z_{1} & Z_{1}^{T} E_{2}^{T} & Z_{1}^{T} E_{2}^{T} \\
0 & E_{1} Y_{1} & E_{2} Z_{1} & -\varepsilon I & 0 \\
0 & E_{1} Y_{1} & E_{2} Z_{1} & 0 & -\varepsilon I
\end{array}\right]
$$

$<0$

where

$$
\Xi_{1}=-\Psi_{0} Z_{1} \Psi_{0}^{T}+\varepsilon H_{2} H_{2}^{T}
$$

and

$$
\Xi_{2}=Y_{1} A^{T}+A Y_{1}+\varepsilon H_{1} H_{1}^{T}
$$




\subsection{Robust control}

Using the analysis so far in this paper we can now establish the following results.

\subsubsection{Discrete processes.}

Theorem 13. Suppose that a control law of the form (18) is applied to a discrete linear repetitive process described by (30) with uncertainty modeled by (32). Then the resulting controlled process is stable along the pass if $\exists$ matrices $P>0$, and $Q>0$, such that

$$
\begin{aligned}
\mathbb{Q} & +(\widetilde{A}+\widetilde{B} \hat{K}+\Delta \widetilde{A}+\Delta \widetilde{B} \hat{K})^{T} \\
& \times P(\widetilde{A}+\widetilde{B} \hat{K}+\Delta \widetilde{A}+\Delta \widetilde{B} \hat{K})<0
\end{aligned}
$$

If this condition holds, a stabilizing control law matrix is given by

$$
K=N Y^{-1} .
$$

\subsubsection{Differential processes.}

Using Theorem 12 we have the following result.

Theorem 15. Suppose that a control law of the form (19) is applied to a differential linear repetitive process described by (31) with uncertainty modeled by (32). Then the resulting controlled process is stable along the pass if, $\exists$ a scalar $\varepsilon>0$, and matrices $Y_{1}>0, Z_{1}>0, N_{1}$, and $N_{2}$, such that

$\left[\begin{array}{ccccc}\Omega_{11} & \star & \star & \star & \star \\ \Omega_{21} & \Omega_{22} & \star & \star & \star \\ Z_{1} \hat{D}_{0}^{T}+N_{2}^{T} \hat{D} & Z_{1} \hat{B}_{0}^{T}+N_{2}^{T} B^{T} & -Z_{1} & \star & \star \\ 0 & E_{1} Y_{1}+E_{3} N_{1} & 0 & -\varepsilon I & \star \\ 0 & 0 & E_{2} Z_{1}+E_{3} N_{2} & 0 & -\varepsilon I\end{array}\right]<0$

Theorem 14. Suppose that a control law of the form (18) is applied to a discrete linear repetitive process described by (30) with uncertainty modeled by (32). Then the resulting controlled process is stable along the pass if $\exists$ a scalar $\varepsilon>0$, and matrices $Y>0, Z>0$, and $N$, such that

$$
\begin{gathered}
{\left[\begin{array}{cccc}
-\Psi Y \Psi^{T} & * & * & * \\
\hat{Y} \hat{A}^{T}+\hat{N}^{T} \hat{B}^{T} & \mathbb{Z} & * & * \\
\varepsilon \hat{H}^{T} & 0 & -\varepsilon I & * \\
0 & \hat{E}_{1} \hat{Y}+\hat{E}_{2} \hat{N} & 0 & -\varepsilon I
\end{array}\right]} \\
<0
\end{gathered}
$$

where

$$
\begin{aligned}
\hat{H} & =\left[\begin{array}{ll}
\hat{H}_{1} & \hat{H}_{2}
\end{array}\right], \quad \hat{E}_{1}=\operatorname{diag}\left(E_{1}, E_{1}\right) \\
\hat{E}_{2} & =\operatorname{diag}\left(E_{2}, E_{2}\right), \quad \hat{A}=\left[\begin{array}{ll}
\hat{A}_{1} & \hat{A}_{2}
\end{array}\right] \\
\hat{B} & =\left[\begin{array}{ll}
\hat{B}_{1} & \hat{B}_{2}
\end{array}\right] \\
\mathbb{Z} & =\left[\begin{array}{cc}
-Y+Z & 0 \\
0 & -Z
\end{array}\right] \\
\hat{Y} & =\left[\begin{array}{ll}
Y & 0 \\
0 & Y
\end{array}\right], \quad \hat{N}=\left[\begin{array}{ll}
N & 0 \\
0 & N
\end{array}\right]
\end{aligned}
$$

where

$$
\begin{aligned}
& \Omega_{11}=-\Psi_{0} Z_{1} \Psi_{0}^{T}+2 \varepsilon H_{2} H_{2}^{T} \\
& \Omega_{21}=Y_{1} \hat{C}^{T}+N_{1}^{T} \hat{D}+2 \varepsilon H_{1} H_{2}^{T} \\
& \Omega_{22}=Y_{1} A^{T}+A Y_{1}+N_{1}^{T} B^{T}+B N_{1}+2 \varepsilon H_{1} H_{1}^{T}
\end{aligned}
$$

If this condition holds, a stabilizing control law matrix is given by

$$
\begin{aligned}
& K_{1}=N_{1} Y_{1}^{-1} \\
& K_{2}=N_{2} Z_{1}^{-1}
\end{aligned}
$$

\section{CONCLUSIONS}

This paper has developed new results on stability and stabilization of discrete and differential linear repetitive processes whose dynamics are second-order in the pass-to-pass direction, with particular attention to avoiding numerical ill-conditioning. The resulting stability conditions and control law design algorithms are LMI based. The core feature is that the algorithms developed do not require the inversion of a possibly ill-conditioned matrix. Also the analysis has been extended to the case when there is uncertainty associated with the process model. Further work consists amongst others, of attempting to use these results to design iterative learning control schemes for second-order illconditioned 1D linear systems, as frequently arise in 
electro-mechanical systems. Note also that all results here can be generalized to higher order processes which are related to so-called non-unit memory linear repetitive processes, which find application in modeling coal mining systems.

The results in this paper, and the methods used to derive them, can also be extended to the case when the along the pass dynamics are second-order as, for example, in the following discrete state-space model

$$
\begin{aligned}
A_{0} x_{k+1}(p+2)= & A_{1} x_{k+1}(p+1)+A_{1} x_{k+1}(p) \\
& +B u_{k+1}(p)+B_{00} y_{k}(p) \\
y_{k+1}(p)= & C_{1} x_{k+1}(p)+D_{1} u_{k+1}(p) \\
& +D_{00} y_{k}(p)
\end{aligned}
$$

where the matrix $A_{0}$ is nonsingular but possibly illconditioned. Such models open up other application areas, such as the development of iterative learning control schemes for descriptor first or the second-order systems. This would, however, require the use of only output feedback control as the state vector here is much harder to recover and the special singular observer must be used [16].

\section{REFERENCES}

1. Inman, D., Vibration with Control Measurement and Stability, Prentice-Hall, New Jersey, NJ (1989).

2. Steffen, P. and R. Rabenstein, "Implicit discretization of linear partial differential equations and repetitive processes," NDS09 6th Int. Workshop Multidimens. (nD) Syst., Thessaloniki, Greece (2009).

3. Datta, B. and D. Sarkissian, "Feedback control in distributed parameter gyroscopic systems A solution of the partial eigenvalue assignment problem," Mech. Syst. Signal Process., Vol. 16, No. 1, pp. 3-17 (2001).

4. Datta, B. and D. Sarkissian, "Theory and computations of some inverse eigenvalue problems for the quadratic pencil," Contemp. Math., Vol. 280, pp. 221-240 (2001).

5. Chahlaoui, Y., D. Lemonnier, A. Vandendorpe, and P. V. Dooren, "Second-order balanced truncation," Linear Alg. Appl., Vol. 415, No. 2-3, pp. 373-384 (2006).
6. Henrion, D., M. Sebek, and V. Kucera, "Robust pole placement for second-order systems: an lmi approach," Proc. IFAC Symp. Robot. Control Design, Barcelona, Spain (2002).

7. Ram, Y. M. and S. Elhay, "Pole assignmnet in vibrating systems with multi-input control," J. Sound Vibr., Vol. 230, pp. 101-119 (2001).

8. Cichy, B. P., Augusta, E. Rogers, K. Gałkowski, and Z. Hurák, "On the control of distributed parameter systems using a multidimensional systems setting," Mech. Syst. Signal Process., Vol. 22, pp. 1566-1581 (2008).

9. Rogers, E. and D. H. Owens, Stability Analysis for Linear Repetitive Processes, Ser. Lecture Notes in Control and Information Sciences, Vol. 175, Springer (1992).

10. Rogers, E., K. Gałkowski, and D. H. Owens, Control Systems Theory and Applications for Linear Repetitive Processes, Ser. Lecture Notes in Control and Information Sciences, Vol. 349, Springer, Berlin, Germany (2007).

11. Amann, N., D. H. Owens, and E. Rogers, "Predictive optimal iterative learning control," Int. J. Control, Vol. 69, No. 2, pp. 203-226 (1998).

12. Paszke, W., Analysis and Synthesis of Multidimensional System Classes Using Linear Matrix Inequality Methods, ser. Lecture Notes in Control and Computer Science, Vol. 8, University of Zielona Góra Press, Zielona Góra, Poland (2005).

13. Xu, S. and J. Lam, Robust Control and Filtering of Singular Systems, Lecture Notes in Control and Information Sciences, Vol. 332, Springer (2008).

14. Boyd, S., L. E. Ghaoui, E. Feron, and V. Balakrishnan, Linear Matrix Inequalities in System and Control Theory, Ser. SIAM Studies in Applied and Numerical Mathematics, Vol. 15, SIAM, Philadelphia, U.S.A. (1994).

15. Khargonekar, P. P., I. R. Petersen, and K. Zhou, "Robust stabilization of uncertain linear systems Quadratic stabilizability and $\mathcal{H}_{\infty}$ control theory," IEEE Trans. Autom. Control, Vol. 35, No. 3, pp. 356-361 (1990).

16. Lam, J., Z. Shu, S. Xu, and E. Boukas, "Robust $H_{\infty}$ control of descriptor discretetime Markovian jump systems," Int. J. Control, Vol. 80, No. 3, pp. 374-385 (2007). 